\title{
Impact of clinical pharmacist managed patient counselling on the knowledge and adherence to antiepileptic drug therapy
}

\author{
Dilip Chandrasekhar, Swetha P. Mohanlal, Abel C. Mathew*, P.K Muhammed Hashik \\ Department of Pharmacy Practice, Al Shifa College of Pharmacy, Perinthalmanna, Kerala, India
}

\section{A R T I C L E I N F O}

\section{Keywords:}

Medication adherence

Counselling

Epilepsy

Healthcare service

\begin{abstract}
A B S T R A C T
Objectives: To evaluate the medication adherence behaviour and knowledge of epilepsy patients before patient counselling and to monitor and evaluate the outcome of clnical pharmacist mediated patient counselling. Methodology: A prospective interventional study with one-year duration was conducted among 100 epileptic inpatients of the neurology department in a south Indian tertiary care hospital. Clinical pharmacy students mediated education was provided to the patients and bystanders, and its effect on medication adherence and patient beliefs was assessed through medication adherence scale (MMAS-4) and an internally validated beliefs about medication survey form.

Results: Before the intervention, $40 \%$ of patients showed high adherence to antiepileptic drug (AED) therapy followed by $27 \%$ patients with a medium level of adherence and 33\% of patients exhibited a low level of adherence. After the intervention, $62 \%$ of patients showed high adherence followed by $20 \%$ of patients with medium adherence and the remaining 18\% exhibited a low level of adherence to AED therapy. Medication belief assessment after intervention also showed improving trends $(P \leq 0.05)$. The study defined that the overall improvement in medication adherence and knowledge of patients was found to be statistically significant after the intervention.

Conclusion: The study concluded that low adherence to the medications and lack of knowledge about the disease was the major reasons for the unsuccessful drug treatment which can be cleared up to a satisfying extent by the use of clinical pharmacist intervention.
\end{abstract}

\section{Introduction}

Epilepsy is one of the most common and widespread neurological disorders. It has a worldwide median incidence of 50.4 cases per one lakh people a year (interquartile range [IQR]: 33.6-75.6). The median incidence of epilepsy in high-income countries is 45.0 cases per one lakh people a year (IQR: 30.3-66.7) and 81.7 cases (IQR: 28.0-239.5) per one lakh people a year in low and middle-income countries. ${ }^{1}$ With a rough estimate of $1 \%$ as the epilepsy prevalence, there are more than 12 million persons living with epilepsy in India, which contributes to almost one-sixth of the total global burden. ${ }^{2}$

WHO defines medication adherence as "the degree to which the person's behaviour corresponds with agreed recommendations from a health care provider". Treatment progress has a great dependency on medication adherence. Reasons for non-adherence are sophisticated and multi-layered. ${ }^{3}$ Patients can unknowingly fail to adhere to their prescribed medications through forgetfulness, misunderstanding, or due to uncertainty about physician's instructions, or intentionally due to their expectations of management, side-effects, and lifestyle choice. AED treatment fails often due to inadequacy of patients to stick with their prescribed regimen. Scanty medication adherence is one of the keystone reasons for poor epilepsy management and negative outcome. This subsequently increases the overall healthcare costs and financial burden on the society along with substantial worsening of seizure and death. Patients who are candidates for repeated adherence issues have frequent hospitalizations with extended stay periods, recurrent emergency department visits and continuous absence from work or school due to seizure effects or anxiety emerging from out of fear of seizure occurrence. More than half of epilepsy patients have poor seizure control due to non-adherence to medication. Non-adherence leads to considerable morbidity, mortality, and avoidable health care costs. ${ }^{2}$

Almost $30 \%$ of people suffering from seizure disorders do not achieve full seizure control even with the best currently available treatment regimen. ${ }^{3}$ So adherence to medication is crucial in the prophylaxis and reducing seizure frequency and their cumulative impact on the quality of life. Current estimates of non-adherence in epilepsy

\footnotetext{
* Corresponding author. Department of Pharmacy Practice, Al-Shifa College of Pharmacy, Poonthavanam P.O, Perinthalmanna, 679325, Kerala, India.

E-mail address: abelcmathew@gmail.com (A.C. Mathew).
} 
are similar to those in other chronic illness and range from 30 to 50\%. This reduces the benefit that could be gained from the medication. ${ }^{5}$

A recent review of methods to improve adherence to anti-epileptic prescriptions suggests three fundamental sorts of interventions as educational, behavioural, and mixed interventions. ${ }^{6}$ The supposition for educational mediation is that patients who comprehend their ailment and its treatment will be increasingly educated, have more control, and be bound to hold fast to treatment. ${ }^{7}$ This is especially significant given the evidence of absence of awareness about the ailments and indications for AEDs among patients with epilepsy, and their requirements for information and counselling. ${ }^{8,9}$ However, published evidence on the effectiveness of this strategy revealed blended results. ${ }^{6}$

Previous proof proposes that pharmacists with knowledge about therapeutic management of chronic illnesses, and training in health education could endow positively toward medication adherence to various long term therapies. ${ }^{10,11}$ A systematic review which analyses five studies also suggests that clinical services offered by pharmacists have a positive impact on knowledge, medication adherence and quality of life of patients with epilepsy. ${ }^{12}$

This prospective interventional study was carried out for a period of one year among the epilepsy patients in the neurology inpatient department of a multispecialty hospital in Perinthalmanna. This is widespread in chronic disease and is a major problem facing medical practice. The primary objective of the study is to evaluate the medication adherence behaviour of epilepsy patients and also monitor the outcome of patient counselling. Moreover, through patient counselling, the patient will be in a good rapport with the pharmacist thereby increasing medication adherence. The study anticipated improving the knowledge and medication adherence behaviour of epilepsy patients through patient counselling.

\section{Methodology}

\subsection{Sample and setting}

The study was carried out in a 500 bedded super speciality tertiary level referral hospital situated in Kerala, the southern part of India. A prospective interventional study carried out among the inpatients of the neurology department to improve the knowledge and medication adherence behaviour of epilepsy patients through patient counselling over 11 months commenced from October 2016 to August 2017. The patients who were diagnosed with epilepsy for at least one year, aged above 18 years, the patients who were treated for epilepsy with at least one antiepileptic drug, subjects who are willing to participate in the study and without other severe comorbidities were included in the study. Pregnant patients, patients of non-Indian origin and patients who provide incomplete information about drug use were excluded from the study. Simple random sampling technique was adopted for drawing patients to the study. A pilot study indicated that a sample size of 30 subjects in each group was sufficient. The size was estimated based on an expected adherence difference of within $25 \%$ at a significance level of $5 \%$.

\subsection{Intervention}

The educational interview involved a 30 -min structured verbal faceto-face interview conducted by a group of two researchers who were clinical pharmacy students individually and privately, in the counselling room at the time of discharge. All patients were educated according to the standard hospital guidelines for patient education and counselling.

The instructive content was separated into two sections. The first part included education about the medical aspects of their disease; epilepsy while the second part included information about AEDs, the importance of adherence, tips to improve the beliefs about medications. Handouts that contained the content of the educational interview were written in Malayalam and English and were introduced in a "question and answer" format, and given after the interview. This leaflet included instructive elements about different aspects of epilepsy including the definition of epilepsy, causes, types, description of seizure generation, diagnostic methods, information about AEDs; and information about the importance of adherence and the consequences of non-adherence and negative beliefs. The interviewers talked methods to incorporate AED administration into their day to day lives and to transform their everyday schedules into signals to take their AEDs. The most widely recognized prompt was meal times.

\subsection{Measurement}

A pharmacist documentation form was designed to collect the information necessary for the study. The form consisted mainly of three modules. The first module included patient demographics and therapy details which included name, age, marital status, place of residence, monthly income, educational qualification, and employment status of the patient. Patient-related characteristics like medical record number, date of admission, comorbid conditions etc., and disease-related aspects like questions about the family history of epilepsy and type of seizure and finally medications and type of therapy receiving. The second module consisted of 4-item Morisky Medication Adherence Scale (MMAS-4) a self-reporting tool which assesses the patient's adherence level to AED therapy through close-ended questions with a score. A score of 0 demonstrates high adherence; a score of 1 or 2 shows intermediate or medium adherence, and a score of 3 or 4 indicates low adherence.

The third module included beliefs about medication survey form. The beliefs about medication survey form is a valid and reliable instrument for evaluating patients' beliefs about medicines in primary healthcare based on literature. ${ }^{13}$ The questionnaire was a self-designed internally validated survey form, divided into two sections which comprised of 10 questions in each section and a 5-point Likert scale was employed to record the response to each statement (strongly disagree, disagree, neither agree or disagree, agree and strongly agree) measuring beliefs about medicines in general and beliefs about AEDs in particular. The general section deals with more general beliefs about medicines such as overuse, harm and benefit beliefs etc., while the specific section deals with concerns and necessity related to AEDs. The answers were scored from 1 (strongly disagree) to 5 (strongly agree). Higher scores in sections were considered as strong negative beliefs about the evaluated criteria in the scale. A score higher than $75 \%$ in every section was considered to be strongly associated with the assessed negative belief.

\subsection{Data collection}

The patients were selected based on the inclusion and exclusion criteria. During ward rounds, each patient was monitored and the data relevant to the study were collected using a data collection form. The patient's data relevant to the study were collected employing interviews with the patients and or their bystanders, by using a questionnaire of known reliability and validity to assess treatment adherence and beliefs about medicines were also measured. Only completed questionnaires were included in the analysis. The score of questionnaires was calculated. The patients or bystanders were provided with counselling aids verbally along with the information leaflet regarding disease and follow-up. The same knowledge questionnaires were used for the follow up after 8 weeks. Based on the score obtained from the MMAS scale and medication belief assessment, adherence was calculated.

\subsection{Ethical consideration}

This study was approved by the institutional ethics committee as per letter no: KAS/ADMN/AC/EC/141/2016 and an official consent were 
also obtained to perform the study. A detailed explanation of the intended study was given to the patient and written consent was obtained from each patient.

\subsection{Statistical analysis}

The entire data collected were analyzed using the appropriate statistical method in consultation with the statistician. Chi-square test was used for comparing the knowledge and adherence pattern of patients before and after the intervention.

\section{Results}

The study is mainly focused on epilepsy patients. The patients were selected from the neurology department of a multispecialty hospital to assess the impact of clinical pharmacist managed patient counselling on the knowledge and adherence to antiepileptic drug therapy. A total of 107 diagnosed cases of epilepsy patients were found eligible for the study. But only 100 patients who have completed the follow-up were included in the study. The study included $56 \%$ of female patients and $44 \%$ male patients. $28 \%$ of patients came under the age category of $18-27$ years. $53 \%$ of them were suffering from focal seizures while $64 \%$ followed monotherapy. Other demographic and clinical characters of respondents are depicted in Table 1.

Table 1

Demographics and clinical characteristics of the study population.

\begin{tabular}{|c|c|c|}
\hline Characteristic & $\mathrm{N}$ & $\%$ \\
\hline \multicolumn{3}{|l|}{ Gender } \\
\hline Male & 44 & $44 \%$ \\
\hline Female & 56 & $56 \%$ \\
\hline \multicolumn{3}{|l|}{ Age } \\
\hline $18-27$ & 28 & $28 \%$ \\
\hline $28-37$ & 25 & $25 \%$ \\
\hline $38-47$ & 15 & $15 \%$ \\
\hline $48-57$ & 12 & $12 \%$ \\
\hline $58-67$ & 11 & $11 \%$ \\
\hline$>67$ & 09 & $09 \%$ \\
\hline \multicolumn{3}{|l|}{ Education } \\
\hline Illiterate & 12 & $12 \%$ \\
\hline Primary $(1-10)$ & 28 & $28 \%$ \\
\hline Secondary (Plus one \& plus Two) & 24 & $24 \%$ \\
\hline College (Diploma, Undergraduate \& Postgraduate) & 36 & $36 \%$ \\
\hline \multicolumn{3}{|l|}{ Marital status } \\
\hline Married & 54 & $54 \%$ \\
\hline Single & 25 & $25 \%$ \\
\hline Divorced & 5 & $5 \%$ \\
\hline Widow/widower & 16 & $16 \%$ \\
\hline \multicolumn{3}{|l|}{ Residence } \\
\hline Urban & 49 & $49 \%$ \\
\hline Rural & 51 & $51 \%$ \\
\hline \multicolumn{3}{|l|}{ Employment status of patients } \\
\hline Employed & 41 & $41 \%$ \\
\hline Self employed & 32 & $32 \%$ \\
\hline Unemployed & 27 & $27 \%$ \\
\hline \multicolumn{3}{|l|}{ Monthly income of patients (in Indian rupees) } \\
\hline$<10000(<\sim 140$ USD $)$ & 46 & $46 \%$ \\
\hline $10000-30000(\sim 140-430$ USD $)$ & 31 & $31 \%$ \\
\hline$>30000(>\sim 430$ USD $)$ & 23 & $23 \%$ \\
\hline \multicolumn{3}{|l|}{ Family history of epilepsy } \\
\hline Positive family history & 39 & $39 \%$ \\
\hline Negative family history & 61 & $61 \%$ \\
\hline \multicolumn{3}{|l|}{ Comorbidities } \\
\hline Patients with comorbidity & 45 & $45 \%$ \\
\hline Patients without comorbidity & 55 & $55 \%$ \\
\hline \multicolumn{3}{|l|}{ Seizure type } \\
\hline Focal & 53 & $53 \%$ \\
\hline Generalized & 35 & $35 \%$ \\
\hline Multiple types & 12 & $12 \%$ \\
\hline \multicolumn{3}{|l|}{ Type of therapy } \\
\hline Monotherapy & 64 & $64 \%$ \\
\hline Polytherapy & 36 & $36 \%$ \\
\hline
\end{tabular}

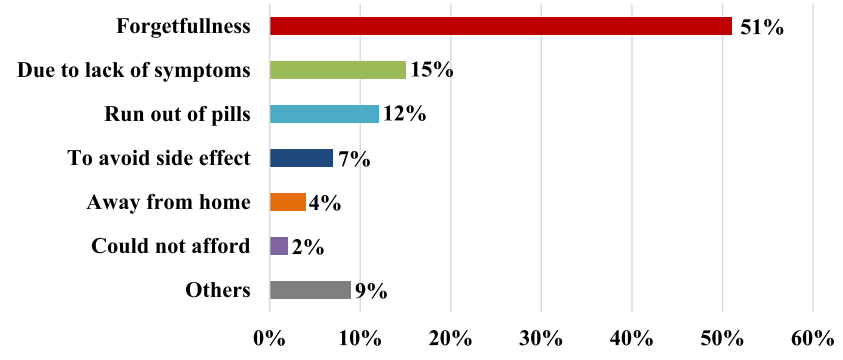

Fig. 1. Reasons for non-adherence.

\subsection{Adherence behaviour of study participants}

Using MMAS-4 patient's adherence level to AED therapy was assessed and it showed that $40 \%$ of patients show high adherence level to AED therapy followed by $27 \%$ of patients shows a medium level of adherence to AED therapy. Remaining $33 \%$ of patients exhibit a low level of adherence to AED therapy before the intervention. Out of 100 patients, the highest number of patients' reason for poor adherence was forgetfulness (51\%). Absence of seizure or lack of symptoms (15\%) was the second most reason for poor adherence. Other reasons for non-adherence was run out of pills, away from home, side effects etc. as a reason for poor adherence. Fig. 1

\subsection{Effect of intervention on patients}

Fig. 2 describes adherence to AED therapy before and after the intervention. Low level of adherence to AED therapy was 33\% $(n=33)$ and $18 \%(\mathrm{n}=18)$ during pre-intervention and post-intervention respectively. The chi-square test showed that the level of adherence to AED therapy was increased in the post-intervention phase when compared with the pre-intervention phase. The study shows a significant reduction in the medium and low level of medication adherence $(\mathrm{P}=0.01)$ and a significant increase in the high level of medication adherence after the intervention $(\mathrm{P}=0.02)$.

Table 2 shows the effect of different factors on adherence level before and after the intervention phase. As age advances, the score obtained by the individuals was also decreasing, indicating that medication adherence was decreasing as age increases. Age and medication adherence were significantly associated $(P<0.05)$. All patients ( $>$ 60years) were non-adherent to their medications. The study shows a significant increase in adherence level after the intervention phase $(P=0.01)$. There was no significant association between gender and adherence rate before and after the intervention $(P>0.05)$. Adherence level was lower in illiterate patients before the intervention phase and which shows significant improvement in the post-intervention phase $(P<0.05)$. Also, statistically significant improvements in adherence patterns in the post-intervention phase were observed among the groups such as married patients, rural residents, employed people, low-income groups, patients without a family history, patients

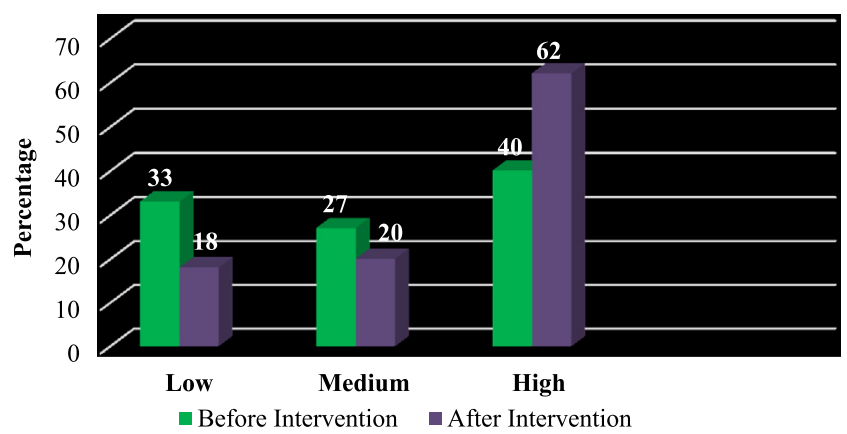

Fig. 2. Adherence to aed therapy before and after intervention. 
Table 2

Demographic factors and adherence.

\begin{tabular}{|c|c|c|c|c|c|c|c|c|}
\hline \multirow[t]{2}{*}{ Variables } & & \multicolumn{3}{|c|}{ Adherence status before the intervention (\%) } & \multicolumn{3}{|c|}{ Adherence status after the intervention (\%) } & \multirow[t]{2}{*}{$P$-value } \\
\hline & & $\mathrm{H}$ & M & $\mathrm{L}$ & $\mathrm{H}$ & M & $\mathrm{L}$ & \\
\hline \multirow[t]{6}{*}{ Age (years) } & $18-27$ & 25 & 3 & 0 & 28 & 0 & 0 & 0.2364 \\
\hline & $28-37$ & 14 & 10 & 1 & 20 & 5 & 0 & 0.1284 \\
\hline & $38-47$ & 2 & 10 & 3 & 8 & 5 & 2 & 0.0649 \\
\hline & $48-57$ & 0 & 3 & 9 & 2 & 7 & 3 & 0.0369 \\
\hline & $58-67$ & 0 & 0 & 11 & 2 & 2 & 7 & 0.0867 \\
\hline & $>67$ & 0 & 0 & 9 & 2 & 1 & 6 & 0.2059 \\
\hline \multirow[t]{2}{*}{ Gender } & Male & 21 & 14 & 21 & 24 & 16 & 16 & 0.6035 \\
\hline & Female & 20 & 12 & 12 & 22 & 13 & 9 & 0.7558 \\
\hline \multirow[t]{4}{*}{ Education } & Illiterate & 0 & 0 & 12 & 3 & 2 & 7 & 0.0373 \\
\hline & Primary & 0 & 8 & 20 & 4 & 15 & 9 & 0.122 \\
\hline & Secondary & 9 & 14 & 1 & 20 & 4 & 0 & 0.0027 \\
\hline & College & 32 & 4 & 0 & 35 & 1 & 0 & 0.3570 \\
\hline \multirow[t]{4}{*}{ Marital status } & Married & 19 & 19 & 16 & 32 & 15 & 7 & 0.0259 \\
\hline & Single & 21 & 4 & 0 & 25 & 0 & 0 & 0.1099 \\
\hline & Divorced & 1 & 1 & 3 & 3 & 2 & 0 & 0.2857 \\
\hline & Widow/Widower & 0 & 2 & 14 & 2 & 3 & 11 & 0.4809 \\
\hline \multirow[t]{2}{*}{ Residence } & Urban & 33 & 15 & 3 & 42 & 8 & 1 & 0.1648 \\
\hline & Rural & 8 & 11 & 30 & 20 & 12 & 17 & 0.0124 \\
\hline \multirow[t]{3}{*}{ Employment status } & Employed & 32 & 9 & 0 & 39 & 2 & 0 & 0.0480 \\
\hline & Self employed & 6 & 14 & 12 & 15 & 12 & 5 & 0.0319 \\
\hline & Unemployed & 3 & 3 & 21 & 8 & 6 & 13 & 0.0899 \\
\hline \multirow[t]{3}{*}{ Monthly income } & $<10000$ & 4 & 16 & 26 & 15 & 16 & 15 & 0.0095 \\
\hline & $10000-30000$ & 16 & 10 & 5 & 25 & 4 & 2 & 0.0668 \\
\hline & $>30000$ & 21 & 0 & 2 & 22 & 0 & 1 & 0.6707 \\
\hline \multirow[t]{2}{*}{ Family history } & Yes & 9 & 7 & 23 & 16 & 9 & 14 & 0.1108 \\
\hline & No & 32 & 10 & 19 & 46 & 11 & 4 & 0.0021 \\
\hline \multirow[t]{2}{*}{ Comorbidity } & Yes & 5 & 12 & 28 & 15 & 13 & 17 & 0.021 \\
\hline & No & 36 & 14 & 5 & 47 & 7 & 1 & 0.0531 \\
\hline \multirow[t]{2}{*}{ Type of therapy } & Monotherapy & 39 & 16 & 9 & 53 & 7 & 4 & 0.0226 \\
\hline & Polytherapy & 2 & 10 & 24 & 9 & 18 & 20 & 0.0581 \\
\hline
\end{tabular}

Abbreviations used: H; High, M; Medium, L; Low.

with comorbidities and patients on monotherapy (Table 2).

Beliefs about medication survey form was used to assess the general beliefs of patients to AED therapy, before and after the intervention, which assess perceptions about AEDs and medications in general. As shown in Fig. 3, the necessity of medication was significantly increased in the post-intervention phase $47 \%(n=47)$ when compared with the pre-intervention phase $(35 \%)(\mathrm{P}=0.02)$. There was a slight decrease in the concerns about the potential side effect in the post-intervention phase $44 \%(n=44)$ when compared with the pre-intervention phase (56\%) $(\mathrm{P}>0.05)$. There was a significant improvement in the overuse, harm-benefit concerns in the post-intervention phase, $(P=0.01)$ and $(P=0.03)$ respectively.

\section{Discussion}

The study assessed the impact of patient counselling on patients' knowledge and adherence to antiepileptic drug therapy. A total of 100 diagnosed cases of epilepsy patients were included in this study. Among this $56 \%$ of epilepsy patients were male and $44 \%$ of epilepsy patients

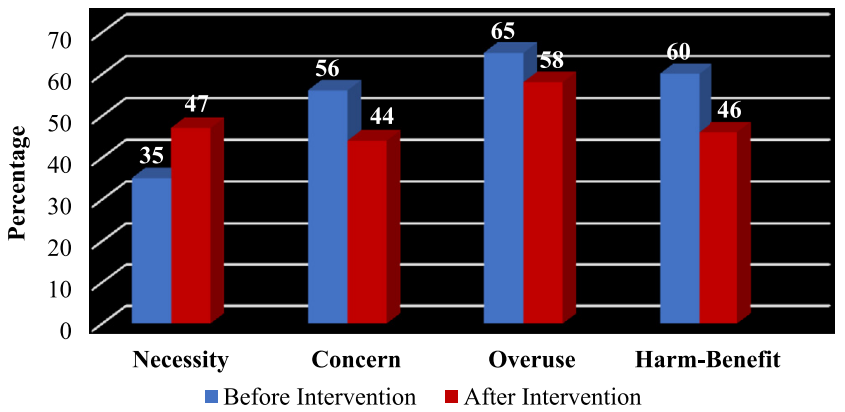

Fig. 3. Beliefs of patients to aed therapy before and after intervention. were female. The reason for higher rates in males may be attributable to the higher frequency of some major etiologies of seizures in men (e. g., cerebrovascular disease, head trauma, alcohol-related seizures) and the study findings were found to be similar with the study conducted by Ranjana Gurumurthy et al. in south India. ${ }^{14}$

Most of the epilepsy patients in this study (64\%) were on AED monotherapy, while $36 \%$ of patients were on AED polytherapy. The result which correlates with Ranjana Gurumurthy et al. ${ }^{14}$ who reported that $53.4 \%$ of epilepsy patients were on AED monotherapy and $46.6 \%$ of patients were on AED polytherapy. These rates were comparatively lower than AlAjmi et al. ${ }^{15}$ study and almost similar with other literature $^{16,17}$

Few studies have used self-reporting measures of adherence to assess adherence to antiepileptic drugs. The validated 4-item Morisky Medication Adherence Scale (MMAS-4) was used to assess the patient's adherence level to AED therapy. Among 100 patients, about $40 \%$ of patients showed high adherence to AED therapy followed by $27 \%$ of patients with medium adherence and $33 \%$ of patients having low adherence to the prescribed regimen before the intervention. These results are comparable with similar studies, ${ }^{15}$ where they used a self-reporting measure of adherence have reported $51.9 \%$ of high adherence. Li et al. $^{18}$ found that self-reported adherence remarkably improved in participants who received intensive health education, repeated reminders, and consultation services, while unchanged adherence levels were reported in the non-intervention group of patients.

Peterson et al. ${ }^{4}$ also reported a positive impact on patient adherence to AEDs among those who received counselling, a pill organizer, and other reminder approaches, measured using blood tests and refill frequencies. In another investigation, there was no huge contrast in adherence to AEDs dependent on distinguishable medication levels or by self-reporting between the intervention and control groups, 1 year following a day-long health educational program. ${ }^{19}$ Dash et al. ${ }^{16}$ analyzed the impact of an organized one-on-one educational program 
administered through an epilepsy nurse over four 30 min sessions. The results revealed that self-reported adherence scores were significantly improved in the intervention group with no huge change in the control group.

These results suggest that the present study had a similar rate of adherence as compared to other studies. There was a significant increase in adherence level was found in the post-intervention phase when compared with the pre-intervention phase $(\mathrm{P}<0.05)$. The current study shows that age and medication adherence was significantly associated. All patients ( $>60$ years) were non-adherent to their medications. This may be due to the fact that elderly patients have more difficulty in following the physician's instructions due to cognitive impairment or other physical deformities such as problems in swallowing tablets, opening pill bottles or handling small tablets and this result was found to be similar with the study conducted by Temesgen Yohannes Hasiso et al. ${ }^{20}$ The study shows that there was no significant association between gender and adherence rate before and after the intervention $(\mathrm{P}>0.05)$ and these findings correlate with Ranjana Gurumurthy et al. ${ }^{14}$

The study shows that patients with higher education show good adherence. As the educational level increases, patient's awareness about the disease and the importance of adhering to medications will increase and it was similar to the result found by Ranjana Gurumurthy et al. ${ }^{14}$

Married patients had a higher level of adherence as compared with single, divorced and widowed patients before and after the intervention. Better adherence in married patients may be due to the support of the partner in adhering to the prescribed medication(s) and the result was found to be similar with the study conducted by Ranjana Gurumurthy et al. ${ }^{14}$ and the study findings were also similar to Wael.M.Gabr et al. ${ }^{21}$

The study identifies a significant improvement in adherence level of divorced and widow/widower patients after the intervention $(\mathrm{P}<0.05)$ Prevalence of non-adherence was higher in patients living in a rural area compared with patients living in urban areas before and after the intervention. This is mainly due to poor patient awareness about epilepsy, the role of antiepileptic medication, and adherence to antiepileptic medication and the study result was found to be similar with the study conducted by Ranjana Gurumurthy et al. ${ }^{14}$

The present study shows that patients with co-morbidity and positive family history of epilepsy show low adherence behaviour to antiepileptic medications before and after the intervention phase and these findings correlate the study conducted by Ranjana Gurumurthy et al. ${ }^{14}$

Monthly income was significantly associated with adherence. Patients with monthly income $<10000$ rupees $(\sim 140$ USD) shows low adherence to antiepileptic drug therapy and this study result was found to be similar to the study conducted by Ogboi Sonny Johnbull et al. ${ }^{22}$

During the time of baseline assessment reasons to stop/miss, medications were assessed. The major reported reasons for non-adherence were forgetfulness $(51 \%)$. This was supported by a number of studies like Liu et al. ${ }^{18}$ They found that the primary reason for non-adherence was forgetfulness $(69.6 \%)$. Also, this finding goes in line with the study of Johnbull et al., ${ }^{22}$ who reported that in more than $40 \%$ of the patients. Paschal et al. ${ }^{23}$ also reported that forgetfulness was the major reason for non-adherence.

Patient's beliefs about their illness and the effectiveness of medication are predictive of adherence and can prolong life and the understanding of poor use of the drug which can lead to treatment failure all impact favourably on adherence. The beliefs about medication survey form assessed perceptions about AEDs in particular and medications in general. Subscales assess general perceptions of 1) the need for medications, 2) concerns of medications, 3) overuse of medicines by doctors and 4) harm and benefit associated with taking medications. ${ }^{13}$

The necessity of medication was significantly increased in the postintervention phase when compared with the pre-intervention phase $(\mathrm{P}=0.02)$. There was a slight decrease in the concerns about potential side effects in the post-intervention phase when compared with the pre- intervention phase $(\mathrm{P}=0.05)$. When compared with the pre-intervention phase the beliefs about the overuse of medicine was significantly decreased in the post-intervention phase $(P=0.01)$. The scoring of harm - benefit was balanced in the post-intervention phase when compared pre-intervention phase $(\mathrm{P}=0.03)$ and the result was found to be similar with the study conducted by Wael.M.Gabr et al., ${ }^{15}$ who reported that patient's beliefs and adherence were associated.

The majority of the patients reported that their epilepsy was not controlled completely after starting their treatment. Therefore, health care professionals must spend sufficient time in educating the patients to reinforce and encourage positive beliefs and discourage negative beliefs to increase patients' medication adherence level. The educational topics should include the potential consequences of poor adherence, the necessity for AED therapy and the safety profile of AED. This study is one of the very few studies that has examined the efficacy of pharmacist-led educational interview in patients with epilepsy. ${ }^{15,16}$ Particularly, this study was helpful in identifying the importance of clinical pharmacy services in the pharmaceutical care and overall quality of life of epilepsy patients since clinical pharmacy services are in its the infancy stage at Indian health care system. This study outcome will assist with showing the benefit of executing a clinical pharmacistled educational intervention at neurology department settings to improve self-care practices and clinical outcomes among Indian epileptic patients.

This study has some confinements such as; self-reporting was the only technique utilized in this study which is subjective in nature and may have underestimated the status of non-adherence when compared to objective measures of non-adherence such as pill count and prescription refills. However, it has been reported that the self-report approach of estimating adherence is a basic, modest and valuable method for distinguishing non-adherence in the clinical setting. Likewise, strict inclusion criteria were applied and response bias was not an issue in this investigation. Care was taken to be non-judgmental during the meeting, yet questioner inclination can't be precluded yet it is accepted to minimally affect study legitimacy.

\section{Conclusion}

The study shows that all patients (age $<60$ years) were non-adherent to their medications. Patients with higher education show good adherence to antiepileptic drugs. Married patients had a higher level of adherence as compared with single, divorced and widowed patients before and after the intervention. Patients with positive family history, comorbidity, living in an urban area, monthly income $<10000$ rupees ( $\sim 140$ USD) shows non-adherence to antiepileptic drugs. Patients on monotherapy were significantly more adherent than patients on polytherapy. The adherence level was significantly increased in the postintervention phase when compared with the pre-intervention phase. Patient's beliefs about their illness and the effectiveness of medication were predictive of adherence and can prolong life. In this study, belief assessment suggested that most of the patients had negative beliefs about the necessity of medications, concerns of medications, overuse of prescribed medications by doctors and harm and benefit associated with taking medications. Thus it can be concluded that provision of proper patient counselling by clinical pharmacist through use of medication aids can improve medication adherence behaviour of the patients and thereby the therapeutic outcome of the medications can be improved and therapeutic failure due to poor adherence can be prevented. Clinical pharmacy services in Indian hospitals are on the budding state. As this study suggests the service of clinical pharmacists can be practised in various healthcare departments and this should be promoted by various medical regulatory bodies.

\section{Funding}

The authors have not received any specific grant for this research 
from any funding agency in the public, commercial or not-for-profit sectors.

\section{Author's contributions}

SPM was responsible for the conceptualization, methodology, data collection and analysis. DC supervised the preparation of the study proposal and conduction of the data collection. ACM and MHPK were involved in data analysis and manuscript writing. DC contributed significantly to revising and improving this article. All authors have critically reviewed and approved the final draft and are responsible for the content and similarity index of the manuscript.

\section{Declaration of competing interest}

The authors report no conflicts of interest in this work.

\section{References}

1. Ngugi AK, Kariuki SM, Bottomley C, Kleinschmidt I, Sander JW, Newton CR. Incidence of epilepsy: a systematic review and meta-analysis. Neurology 2011;77:1005-1012.

2. Gururaj G, Satishchandra P, Amudhan S. Epilepsy in India I: epidemiology and public health. Ann Indian Acad Neurol. 2015;18:263.

3. Sabaté E. Adherence to Long-Term Therapies - Evidence for Action. World Health Organization; 2003 accessed http://apps.who.int/medicinedocs/en/d/Js4883e/, Accessed date: 22 September 2018.

4. Peterson GM, Mclean S, Millingen KS. A randomised trial of strategies to improve patient compliance with anticonvulsant therapy. Epilepsia. 1984;25:412-417.

5. Burger J, Jacobs K, Julyan M, Lubbe M, Cockeran M. Medicine possession ratio as proxy for adherence to antiepileptic drugs: prevalence, associations, and cost implications. Patient Prefer Adherence. 2016:539-547.

6. Al-Aqeel S, Gershuni O, Al-Sabhan J, Hiligsmann M. Strategies for improving adherence to antiepileptic drug treatment in people with epilepsy. Cochrane Database Syst Rev. 2017;2:CD008312

7. Touchette DR, Shapiro NL. Medication compliance, adherence, and persistence: current status of behavioral and educational interventions to improve outcomes. Manag Care Pharm. 2008;14(6 Suppl D):2-10.
8. Couldridge L, Kendall S, March A. A systematic overview-a decade of research. The information and counselling needs of people with epilepsy. Seizure. 2001;10:605-614.

9. Long L, Reeves AL, Moore JL, Roach J, Pickering CT. An assessment of epilepsy patients knowledge of their disorder. Epilepsia. 2000;41:727-731.

10. Faught RE, Weiner JR, Guérin A, Cunnington MC, Duh MS. Impact of nonadherence to antiepileptic drugs on health care utilization and costs: findings from the RANSOM study. Epilepsia. 2009;50:501-509.

11. Lie IA, Hoggen I, Samsonsen C, Brodtkorb E. Treatment non-adherence as a trigger for status epilepticus: an observational, retrospective study based on therapeutic drug monitoring. Epilepsy Res. 2015;113:28-33.

12. Reis TM, Campos MS, Nagai MM, Pereira LR. Contributions of pharmacists in the treatment of epilepsy: a systematic review. Am J Pharm Benefit. 2016;8(3) e55-e60.

13. Ioannis D. Komninos, katerina micheli, theano roumeliotaki and rob home. Adaptation and validation of the beliefs about medicines questionnaire in primary care patients in Greece. Eur J Pers Cent Healthc. 2013;1(1):224-231.

14. Gurumurthy Ranjana, Kulkarni Chanda, Sarma. An evaluation of factors affecting adherence to anti-epileptic drugs in patients with epilepsy: a cross-sectional study. Singap Med J. 2017;58(2):98-102.

15. AlAjmi Refah. The impact of a pharmacist-led educational interview on medication adherence of Saudi patients with epilepsy. Patient Prefer Adherence. 2017;11:959-964.

16. Dash D, Sebastian TM, Aggarwal M, Tripathi M. Impact of health education on drug adherence and self-care in people with epilepsy with low education. Epilepsy Behav. 2015;44:213-217.

17. Sweileh WM, Ihbesheh MS, Jarar IS, et al. Self-reported medication adherence and treatment satisfaction in patients with epilepsy. Epilepsy Behav. 2011;21(3):301-305.

18. Liu J, Liu Z, Ding H, Yang X. Adherence to treatment and influencing factors in a sample of Chinese epilepsy patients. Epileptic Disord. 2013;15:289-294.

19. Ibinda F, Mbuba CK, Kariuki SM, et al. Evaluation of Kilifi epilepsy education programme: a randomized controlled trial. Epilepsia. 2014;55(2):344-352.

20. Temesgen Yohannes Hasiso. Tigestu Alemu Desse. Adherence to treatment and factors affecting adherence of epileptic patients at yirgalem general hospital, southern Ethiopia: a prospective cross-sectional study. PloS One. 2016;11(9):1-12.

21. Gabr Wael M, Shams Mohamed EE. Adherence to medication among outpatient adolescents with epilepsy. Saudi Pharmaceut J. 2015;23:33-40.

22. Johnbull Ogboi Sonny, Farounbi Babajide, Ademola O Adeleye, Ogunrin Olabunmi, Agu P, Uche. Evaluation of factors influencing medication adherence in patients with epilepsy in rural communities of kaduna state, Nigeria. Neurosci Med. 2011;2:299-305.

23. Paschal AM, Hawley SR, Romain TS, et al. Epilepsy patients' perceptions about stigma, education, and awareness: preliminary responses based on a community participatory approach. Epilepsy Behav. 2007;11(3):329-337. 